\title{
Modelo Estocástico para la eficiencia global de los equipos (OEE): Consideraciones prácticas para su utilización
}

\section{Stochastic model for Overall Equipment Effectiveness: practical considerations}

Modèle stochastique pour l'efficacité globale des équipements (OEE) : considérations pratiques

\begin{tabular}{c}
\hline Modelo Estocástico para a eficiência do \\
equipamento global (OEE): considerações \\
práticas
\end{tabular}

Héctor René Álvarez Laverde * Rocío Andrea Sánchez Silva**

Fecha de recepción: 6 de octubre

Fecha de aprobación: 28 de octubre

Pp. 53-86

\footnotetext{
* Ph.D. en Aplicaciones Técnicas e Informáticas de la Estadística, Universidad Politécnica de Cataluña. Magíster en Estadística, Universidad Nacional de Colombia. Especialista en Ingeniería de Producción, Universidad Distrital Francisco José de Caldas. Matemático, Universidad Nacional de Colombia.

** Magíster en Ingeniería de Producción, Universidad Distrital Francisco José de Caldas. Especialista en Estadística, Universidad Nacional de Colombia. Matemático con énfasis en Estadística, Universidad del Tolima.
} 


\section{RESUMEN}

En los entornos de eficiencia de operacional se utiliza una métrica que permite medir la eficiencia global de los equipos y de las líneas de producción (OEE). Esta es una métrica desarrollada en entornos TPM que integra la disponibilidad, el rendimiento y la calidad obtenida en los equipos. Este indicador que es estratégico, que bien utilizado puede ser muy robusto para la planificación y toma decisiones, en general no se utiliza de forma apropiada, porque solo se calcula como KPI de referencia, sin entrar a profundizar es el análisis del comportamiento.

El objetivo de este trabajo es presentar un modelo estocástico para el OEE, que considera los múltiples factores inciertos que afectan al OEE y que ocurren dentro de la operación de un equipo o línea de producción. A partir del modelo se establece una metodología para analizar el OEE desde una perspectiva más amplia, específicamente su variabilidad, permitiendo hacer un análisis más robusto, además se presenta una aplicación práctica del modelo haciendo algunas consideraciones para su uso y toma de decisiones.

\section{Palabras Clave}

Eficiencia, OEE, árbol de perdidas, modelo estocástico.

\section{Abstract}

In environments of operating efficiency, metrics to measure the Overall Efficiency of Equipment (OEE) and production lines is used. This is a metric developed in environments that integrate Total Productive Maintenance (TPM) availability, and the efficiency and quality obtained in the equipment. This indicator that is strategic, and if used in the right way can be very robust for planning and making decisions, in general is not used properly, because only Key Performance Indicators (KPI) are calculated as reference, without going into further analysis of its behavior.

This document presents a stochastic model proposed for the OEE, which allows you to change the paradigm of its analysis; given the fact that the OEE has a random behavior because it is affected by many uncertain factors occurring within the operation of a machine or production line. From the 
proposed model, several practical considerations regarding its use and implementation are made. The main reflection is related to how important it is to analyze the variability of $O E E$, since it is very relevant for the ultimate goal of operational efficiency and decision-making.

\section{KEYWORDS}

Eficiency, overall efficiency of equipment (OEE), loss tree, stochastic model.

\section{RÉSUMÉ}

En termes d'efficacité opérationnelle il s'agira ici d'utiliser une métrique permettant de mesurer l'efficacité globale des équipements et des lignes de production (OEE). Cette métrique, développée dans des environnements TPM, intègre la disponibilité, le rendement et la qualité des équipements. Cet indicateur stratégique, utilisé correctement, devient très efficace pour la planification et la prise de décisions. Cependant, cet indicateur, mesuré uniquement à partir du KPI de référence n'est généralement pas utilisé de manière adéquate pour l'approfondissement de l'analyse du comportement. Nous présentons dans cet article un modèle stochastique proposé pour l'OEE permettant un changement de paradigme de l'analyse. L'OEE connait un comportement aléatoire intrinsèque car il peut être affecté par de multiples facteurs se produisant au sein des opérations, d'un équipement ou d'une ligne de production. À partir du modèle proposé, nous émettrons certaines considérations pratiques pour son usage et application. La considération principale montre l'importance d'analyser la variabilité de l'OEE qui influe largement sur l'objectif final d'efficacité opérationnelle et de prise de décisions.

\section{MOTS CLEFS}

Efficacité, OEE, Schéma des pertes, Modèle stochastique. 


\section{Resumo}

Nos ambientes de eficiência operacional se utiliza uma métrica que permite medir a eficiência global dos equipamentos e das linhas de produção (OEE). Esta é uma métrica desenvolvida em ambientes TPM que integra a disponibilidade, o desempenho e qualidade obtida no equipamento. Este indicador que é estratégico, se bem utilizado pode ser relevante para o planejamento e tomada de decisão, em geral, não é usado corretamente, porque só se calcula como KPI de referência, sem entrar em uma análise mais aprofundada do comportamento.

Aqui se apresenta um modelo estocástico proposto para o OEE, o qual permite mudar o paradigma de suas análises. Dado o facto de que o OEE tem um comportamento aleatório, uma vez que é afetado por muitos fatores incertos que ocorrem dentro do funcionamento de uma máquina ou linha de produção. A partir do modelo proposto, várias considerações práticas da sua utilização e aplicação são feitas. As principal é que é importante analisar a variabilidade do OEE, porque é um ponto muito importante relacionado com objetivo final da eficiência operacional e tomada de decisões.

\section{Palavras-chave}

Eficiência, OEE, árvore de perdas, modelo estocástico. 


\section{Introducción}

uando las empresas se enfrentan al reto de optimizar los procesos y las operaciones, dentro de una visión de la excelencia operacional, el primer objetivo que deben buscar es la reducción de las pérdidas, porque ellas son principalmente la fuente de todas las ineficiencias de las plantas industriales y constituyen en gran parte a la famosa llamada "fabrica oculta". El modelo TPS (Toyota Production Systems) (Womack \& Jones, 1996), sobre el cual están basados todos los modelos de excelencia operacional, plantea que en toda planta existen 16 grandes pérdidas, que deben ser eliminadas o minimizadas. Las pérdidas están clasificadas por tipo dependiendo su naturaleza y las causas que la generan en el proceso. La base de la gestión de la perdidas es el llamado árbol de pérdidas (Stamatis, 2010), el cual permite mapear las pérdidas de una organización, y tener una visión general de su estructura y el comportamiento, y lo más importante, relacionándola con los costos y así tener un indicador más robusto para la toma de decisiones.

El indicador de Eficiencia Global de Equipos (OEE- Overal Eficiency Equipment), que fue propuesto por Nakajima (1988) dentro del entorno del TPM (Total Productive Maintenance), como una métrica para el análisis de eficiencia de la utilización de los recursos dentro de una línea de fabricación. Ella ha permitido a muchas empresas realizar mejoras enfocadas a la optimización y/o eliminación de las pérdidas. La eficiencia global de equipos OEE, de acuerdo como lo definió Nakajima está en términos de la utilización óptima del tiempo que los equipos y los recursos están disponibles para producir, desde el punto de vista conceptual el OEE se define específicamente como el producto de tres indicadores: 


\section{OEE=Disponibilidad $\times$ Rendimiento $\times$ Calidad}

Donde la Disponibilidad es la proporción del tiempo que el proceso es productivo con respecto al tiempo total disponible, por tanto es afectada completamente por las paradas (programadas y no programadas) que tiene el sistema; el Rendimiento es la fracción de la producción real que se produce en el tiempo productivo con respecto a la producción teórica, así el rendimiento es afectada por las reducciones de velocidad y las pequeñas paradas del sistema; y el Índice de Calidad es la fracción de todas las unidades reales producidas que son conformes con respecto a todas las unidades producidas, así el índice de calidad es afectada por todo el tiempo perdido por fabricar componentes no conformes. Dentro del modelo TPM, estos indicadores que componen el OEE se definen en términos de pérdida de tiempo, y se entiende como la utilización máxima de los recursos a través de la utilización máxima del tiempo disponible del sistema productivo (Ljunberg 1998).

Analizando en detalle la dinámica del comportamiento del OEE, está afectada por múltiples factores asociados a los tres elementos que la componen: en la disponibilidad (por todos los tipos y causales de paradas, tanto programadas como no programadas); en el rendimiento (las continuas fluctuaciones y cambios de velocidad, así como el control de las micro paradas, que se presentan las líneas); y en la calidad del producto final (todos los aspectos asociados y causales de no conformidades, los cuales generan una pérdida de tiempo y de materia prima).

Es pertinente señalar que en este artículo se muestran algunos resultados de la tesis de maestría en Ingeniería Industrial desarrollada en la Universidad Distrital Francisco Jose de Caldas (Sánchez, 2015). 


\section{Planteamiento del problema y objetivos}

E

n la práctica actual, como se utiliza el OEE es como un KPI (Key Perfomance Indicator) más de productividad, es un frio número que no es analizado en detalle, ni es desglosado para la toma robusta de decisiones. Gouvea \& Pimheiro (2002), hacer un análisis más amplio de los usos y abusos del OEE y además plantean que dependiendo de como se defina y se interpreten los factores que lo afectan se puede tener una medición diferente que debe ser entendida completamente para su correcta interpretación. Y como consecuencia las posibles acciones de mejora que deben implementarse para tener un mejor desempeño, reflejado en un aumento de eficiencia global.

En general, el OEE es usado e interpretado como un valor promedio, si considerar su variabilidad, se gestiona de una forma completamente determinística y a partir de allí se toman las decisiones, el planteamiento es que si se analiza su dispersión, se puede entrar a comprender su naturaleza aleatoria.

Por tanto, es importante analizar OEE con este enfoque estocástico permitiendo cuantificar las mejoras y la reducción de pérdidas basados no solo con el aumento promedio del OEE, sino también en reducción de dispersión, lo cual se refleja la estandarización de los procesos y de esta forma tener un mayor efecto en la optimización. Por lo tanto el objetivo del presente trabajo es: plantear un modelo estocástico del OEE, que pueda evaluar no solo el comportamiento promedio, sino también la variación, permitiendo obtener un análisis más detallados y robustos de la eficiencia de los equipos. 
También se hacen consideraciones prácticas para el uso del modelo propuesto.

En la literatura técnica no se ha reportado ningún estudio similar que aborde el problema de la variabilidad del OEE y su aplicación práctica.

\section{Estado del arte}

\subsection{Caracterizacion del OEE}

EI OEE como fue propuesto por Nakajima (1988) y definido en la ecuación (1), este fué ampliado por Suzuki (1999) donde toma como referencia el tiempo total disponible del equipo (tiempo calendario), a partir de este se calcula el Tiempo Disponible Neto de Operación -TDN, que excluye el tiempo no disponible planeado, tales como los tiempos de mantenimiento planeado o los tiempos fuera de candelario, este es el tiempo donde se programan los recursos para producir. Al TDN se resta las paradas (programadas y no programadas) obteniéndose el Tiempo Total Operativo (TTO), después se calcula el Tiempo Operativo Real (TOR), excluyen los tiempos perdidos por las pequeñas paradas y reducciones de velocidad del tiempo de operación y finalmente se calcula el Tiempo Operativo Efectivo (TOE) restando los tiempos perdidos por generación de no conformes y mermas de material en proceso, es decir, el tiempo donde se utilizan completamente los recursos del proceso (Figura 2). 
Figura 1. Not.ción de tiempos para OEE

\begin{tabular}{|l|l|}
\hline Tiempo Total Disponible $=$ TTD & \\
\hline Tiempo Disponible Neto $=$ TDN & $\begin{array}{l}\text { Tiempo Planeado No } \\
\text { Operativo }=\text { TNO }\end{array}$ \\
\hline Tiempo Total Operativo $=$ TTO & $\begin{array}{l}\text { Tiempo de } \\
\text { Paradas }=\text { TTP }\end{array}$ \\
\hline Tiempo Operativo Real $=$ TOR & $\begin{array}{l}\text { Tiempo perdido por } \\
\text { Rendimiento }=\text { TPR }\end{array}$ \\
\hline Tiempo Operativo Efectivo = TOE & $\begin{array}{l}\text { Tiempo perdido por } \\
\text { Calidad }=\text { TPC }\end{array}$ \\
\hline
\end{tabular}

Fuente. Elaboración propia de los autores.

De esta forma se puede definir el OEE en términos de fracción de tiempos operativos, de la forma siguiente:

$\mathrm{OEE}=\frac{\text { (Tiempo Operativo Efectivo) }}{\text { (Tiempo Disponible Neto de Operación) }}=$ TOE $/$ TDN

El éxito computacional del OEE depende de la habilidad de recolectar los datos. Si la colección de los datos no es fiable, el valor del OEE calculado puede no reflejar la utilización real del equipo, además cada pérdida clasificada corresponde a un estado del equipo. Esto quiere decir que debe estar definida de antemano la clasificación de las pérdidas, en este sentido cada compañía puede definir estas clasificaciones, pero se recomienda usar el estándar SEMI E10-92 (1992), ella las clasifica en 10 clases de pérdidas, y basada en el desglose del Árbol de Pérdidas (Ljungberg, 1998).

\subsection{Modelo estocástico del OEE}

El primer aspecto que se estudia es la estructura estocástica del OEE. Para esto se analiza la naturaleza aleatoria de algunas de las pérdidas que integran y que determinan el comportamiento del OEE, en particular se analizan la naturaleza de las pérdidas en cuatro situaciones específicas. 
- Pérdida por ocurrencia de Averías. A pesar de que las averías que ocasionan paradas inesperadas de los equipos están asociadas a las políticas de mantenimiento que se establecen en la organización (mantenimiento correctivo, preventivo, basado en el tiempo, basado en las condiciones o basado en la confiabilidad) ellas se presentan en forma incierta; la política de mantenimiento afecta a la probabilidad de ocurrencia de la avería. Por ejemplo si la política de mantenimiento es basada en corrección es posible que se tengan factores causales que agravan la avería, como la falta lubricación, en este caso la probabilidad de ocurrencia de la avería es mucho mayor que en el caso de que se tenga una política de mantenimiento basada en la condición, donde se realizan inspecciones y revisiones periódicas de las condiciones de funcionamiento de los equipos y se realizan acciones de mantenimiento preventivo, aún más cuando el operador tiene rutinas de revisión, el cual permite que de una forma temprana se den cuenta que hace falta de lubricación (mantenimiento autónomo). Otro aspecto que influye en la estocasticidad de la avería es el tiempo de intervención, este depende de la gravedad, de la capacidad técnica y operativa que tienen los técnicos de mantenimiento, para resolver la avería.

- Pérdidas inesperadas por ajustes de proceso. En este caso se genera paradas para realizar ajusten en el proceso, este también es de naturaleza aleatoria porque está asociada a la causa del ajuste. En particular, en el caso que sean por ajustes de parámetros de proceso, existió una evidencia o un síntoma de que los parámetros estaban fuera de control o simplemente empezó a presentar productos no conformes (pérdidas de calidad), dependiendo de la respuesta ante esta incidencia, se generan distintos tipos de pérdidas: Una primera posibilidad es que el operador decida no parar el proceso para hacer ajustes, sino reducir 
la velocidad en este caso se reduce el rendimiento, otra posibilidad es que el operario decida parar el proceso y hacer ajustes, en este caso se genera una pérdida por parada, impactando la disponibilidad, si adicionalmente la parada genera pérdidas por desperdicio de materia prima, se aumenta el efecto. Además de la aleatoriedad de la ocurrencia el tiempo de intervención también es aleatorio.

- Paradas inesperadas por problemas logísticos. En el caso que se presenten paradas por la no disponibilidad de materia prima, o insumos de producción o logísticos, la parada es de naturaleza estocástica, porque ella se presenta en forma imprevista, el causal es una mala planeación o la ineficiencia del proceso o un error humano, en todos los caso se presenta en forma incierta. Aquí se puede incidir en la probabilidad de ocurrencia del evento, por ejemplo si no hay planificación o es muy deficiente para la disponibilidad del abastecimiento de materiales e insumos logísticos, la probabilidad de ocurrencia es muy alta. De la misma forma, la duración de la parada es incierta, porque depende de la capacidad que tienen para resolver la incidencia.

- Paradas programadas. Se podría pensar que las paradas programadas por su naturaleza no son aleatorias, pero no es así. Por ejemplo en los cambios de referencia en los equipos, ya está planificado en el programa de producción que se debe realizar los cambios de una referencia a otra, en tal caso la parada no es inesperada, el comportamiento aleatorio está asociado a la frecuencia con que se hacen los cambios de referencia está asociada a los requerimientos de la demanda, y en este caso la demanda tiene un comportamiento aleatorio. De igual forma, el tipo de cambio va afectar directamente a la duración del cambio y el tiempo de la parada. 
De la misma forma si se analizan en detalle todas las demás componentes y factores que afectan al OEE, todos ellos están afectados por eventos y situaciones aleatorias, por tanto, es coherente pensar en un modelo que describa el comportamiento probabilístico del OEE.

Ya teniendo claro la naturaleza estocástica del OEE, se va a formular el modelo que rige su comportamiento, para esto se van a definir las variables y la notación para la construcción del modelo (figura 1).

TTD = Tiempo Total Disponible (tiempo calendario)

TDN = Tiempo Disponible Neto (tiempo disponible para producir)

TTO = Tiempo Total Operativo (tiempo donde se producen unidades)

TOR = Tiempo Operativo Real (tiempo donde se producen unidades al máximo rendimiento)

TOE $=$ Tiempo Operativo Efectivo (tiempo operativo que se producen unidades buenas)

TPR = Tiempo Perdido por reducción del Rendimiento (reducción velocidad)

TPC = Tiempo Perdido por Calidad (por producir unidades defectuosas)

TTP = Tiempo Total de Paradas (paradas programadas y no programadas).

Considerando esta notación se puede definir el OEE de la siguiente forma:

$$
\begin{aligned}
\text { OEE }=D \times R \times C \\
\text { OEE }=\frac{\text { TOT }}{\text { TDN }} \times \frac{\text { TOR }}{\text { TOT }} \times \frac{\text { TOE }}{\text { TOR }} \\
\text { OEE }=\frac{\text { TOE }}{\text { TDN }}=\frac{\text { Tiempo Operativo Efectivo }}{\text { Tiempo Disponible Neto }}
\end{aligned}
$$


Para expresar todas las componentes en términos de tiempo se debe hacer la conversión de unidades producidas a tiempo perdido (como está definido el rendimiento y el índice de calidad), para hacer esta conversión se utilizan los siguientes términos:

NUP = Número de Unidades Producidas

NUD $=$ Número de Unidades Defectuosas

$\mathrm{TC}=$ Tiempo de Ciclo = Tiempo Teórico empleado para producir una unidad

De esta forma los tiempos perdidos por rendimiento y por problemas de calidad se calculan como:

$$
\begin{gathered}
\mathrm{TPR}=\left(\frac{\mathrm{TOT}}{\mathrm{TC}}-\mathrm{NUP}\right) \times \mathrm{TC}=\mathrm{TOT}-\mathrm{NUP} \times \mathrm{TC} \\
\mathrm{TPC}=(\mathrm{NUP}-\mathrm{NUD}) \times \mathrm{TC}
\end{gathered}
$$

Así el OEE se puede calcular usando los siguientes tiempos:

$$
\begin{gathered}
\text { OEE }=\frac{\text { TDN }- \text { TTP }}{\text { TDN }} \times \frac{\text { TOT }- \text { TPR }}{\text { TOT }} \times \frac{T O R-T P C}{T O R}=\frac{T O E}{T D N} \\
\text { OEE }=\frac{\text { TDN }- \text { TTP }- \text { TPR }- \text { TPC }}{\text { TDN }}
\end{gathered}
$$

El OEE definido de esta forma, es el más utilizado en las áreas operativas para toma de decisiones, este conocido como el OEE operacional, y más comúnmente notado como OEE2 (Suzuki, 1995). Así el OEE es el cociente de dos tiempos que son variables aleatorias, que a su vez cada una es suma de múltiple variables aleatorias, que la componen. Por ejemplo el TTP es la suma de todos los tiempos de paradas, ocurridas durante el periodo de análisis, es por esto que se utiliza el teorema central de límite como supuesto que este tiempo tiene un comportamiento Normal (Roussas, 1997). De esta 
forma, el modelo general supone que el OEE es el cociente de dos variables normales.

Para caracterizar completamente el OEE como variable aleatoria, se debe desglosar la función de densidad de probabilidad, que indica el comportamiento probabilístico de la variable, de esta forma se puede establecer propiedades más robustas de la variable. El comportamiento del cociente de variables aleatoria depende del comportamiento particular tanto del numerador como del denominador y su dependencia estadística.

Al revisar la literatura técnica, Zammori et als (2011) hace un planteamiento de modelar los tiempos con una distribución Beta $(\alpha, \beta)$, partiendo de la aplicación de la distribución Beta en el análisis de proyectos en la optimización del PERT a través de la ruta crítica, éste mismo análisis plantea que la distribución Beta es una familia de variables aleatorias que tiene una gran cobertura de posibilidades para modelar el tiempo. Farnun \& Stanton (1987) quienes formalizaron la aplicación al PERT, consideran que siempre hay un valor mínimo $\alpha$ (optimista) un máximo $\beta$ (pesimista) y un máximo m (más probable).

Debido que los tiempos de pérdidas, son la suma de un número grande de tiempos, se puede considerar los tiempos en el numerador como la son suma de tiempos $\mathrm{T}_{\mathrm{i}}$. Esta es una característica importante, porque una realización (es decir, el valor observado) del tiempo perdido $T_{i}$ que nunca puede ser menor de cero y rara vez puede ser más grande que el valor promedio, por lo tanto el comportamiento de los tiempos perdidos $T_{-}$i son generalmente sesgados al lado derecho y puede ser fácilmente modelado con una distribución Beta con parámetros $\alpha>1$ y $\beta \geq \alpha$. 
De esta manera usando el teorema central del límite se obtiene:

$$
\mathrm{T} \sim \sum \mathrm{T}_{\mathrm{i}} \sim \mathrm{N}\left(\mu, \sigma^{2}\right) \text { Donde } \mu=\sum \mu_{\mathrm{i}} \quad \text { y } \sigma^{2}=\sum \sigma_{\mathrm{i}}^{2}
$$

Considerando las condiciones y propiedades del teorema central del límite, parece razonable considerar la distribución de los tiempos netos de operación como una variable aleatoria, partiendo del tiempo de pérdidas $\mathrm{T}_{\mathrm{i}}$. Es importante resaltar que aunque el supuesto del teorema central del límite dice que las $n$ variables $T_{i}$ debe tender a infinito. Law \& Kelton (1997) demuestra que con solo $n \geq 7$ asegura la aplicabilidad del TCL, esta condición es suficientemente satisfecha porque generalmente el número de pérdidas es bastante grande en un periodo de un turno o día de operación.

Para el modelo definido en este documento, se considera que cada uno de los tiempos que componen tanto el numerador como el denominador, tienen una distribución Weibull. De la misma forma se puede suponer la hipótesis del teorema central del límite, que la suma tiene un comportamiento Normal (porque igualmente se tiene para cualquier tipo de distribución). En este punto se puede entrar a formalizar el modelo propuesto.

Bajo los anteriores supuestos, se considera que $\mathrm{X}=\mathrm{TOE} \sim \mathrm{N}\left(\mu_{\mathrm{X}^{\prime}} \sigma_{\mathrm{X}}{ }^{2}\right)$ y $\mathrm{Y}=\mathrm{TDN} \sim \mathrm{N}\left(\mu_{\mathrm{Y}^{\prime}} \sigma_{\mathrm{Y}}{ }^{2}\right)$ por lo tanto se puede reescribir que $\mathrm{OEE}=\mathrm{U}=\mathrm{X} / \mathrm{Y}$ donde en general su comportamiento probabilístico no es normal, en este caso hay un supuesto muy importante a considerar y es la independencia entre las variables $X$ y $Y$, por esta razón se consideran dos casos:

- Cuando X y Y son variables independientes. Este primer caso que es el más sencillo, pero que no es el caso que se está tratando. Roussas (1997) describe la distribución 
del cociente a través de un modelos probabilístico Cauchy. Como caso particular, sean las variables aleatorias $\mathrm{X} \sim \mathrm{N}\left(0, \sigma_{X}{ }^{2}\right)$ y $\mathrm{Y} \sim \mathrm{N}\left(0, \sigma_{Y}{ }^{2}\right)$ independientes, el análisis no pierde generalidad al suponer que las variables tiene media cero.

La función de densidad de probabilidad está dada por:

$$
f_{U}(u)=\frac{1}{\pi \sigma_{X} \sigma_{Y}} \frac{1}{2\left[\frac{1}{2 \sigma_{Y}^{2}}+\frac{u^{2}}{2 \sigma_{X}^{2}}\right]}=\frac{1}{\pi}\left[\frac{\sigma_{X} \sigma_{Y}}{u^{2} \sigma_{Y}^{2}+\sigma_{X}^{2}}\right]=\frac{1}{\pi}\left[\frac{\frac{\sigma_{X}}{\sigma_{Y}}}{u^{2}+\frac{\sigma_{X}^{2}}{\sigma_{Y}^{2}}}\right]
$$

Por lo tanto la función de densidad de probabilidad de $U$ corresponde a una distribución de Cauchy

$$
f_{U}(u)=\frac{1}{\pi}\left[\frac{\left(\frac{\sigma_{\mathrm{X}}}{\sigma_{\mathrm{Y}}}\right)}{\mathrm{u}^{2}+\left(\frac{\sigma_{\mathrm{X}}}{\sigma_{\mathrm{Y}}}\right)^{2}}\right] \quad \text { con } \quad \mathrm{u} \in \mathbb{R}
$$

Esta corresponde a una distribución Cauchi con parámetros $\alpha=\frac{\sigma_{X}}{\sigma_{Y}}$ y $\beta=0$ (Roussas, 1987).

- Cuando X y Y no son variables independientes. Este caso es más general y aplicable a la situación de análisis de OEE, las variables $X$ y $Y$ son dependientes, porque en la ecuación (5) se observa que el numerador depende del denominador (El numerador está definido en términos del denominador).

Como $X=T O E=T D N-T T P-T P R-T P C \sim N\left(\mu_{x^{\prime}} \sigma_{x}^{2}\right)$ y

$\mathrm{Y}=\mathrm{TDN} \sim \mathrm{N}\left(\mu_{\mathrm{Y}}, \sigma_{\mathrm{Y}}{ }^{2}\right)$, para derivar la función de probabilidad se define la variable $Z$ como $Z=T T P+T P R+T P C$ por lo tanto, $X=Y-Z$, de esta manera el numerador $X=T O E$ es función del denominador $Y=T D N$, dónde no son independientes. Por lo tanto para el $O E E=X / Y$ se tiene que $0 \leq X / Y \leq 1$ y $Y=X+Z$, entonces $U=O E E$, nunca será mayor 1 . 
De la misma forma se puede considerar que se tiene $\mu_{Y}>\mu_{X^{\prime}}$ debido a que $Y=X+Z$, la función de densidad de probabilidad (fdp) tiene un desvanecimiento (cola con alta asintoticidad) cercano a uno, esto permite considerar una aproximación de la fdp real de OEE a través de una función truncada de $f_{u}(u)$, expresada de la siguiente forma:

$$
f_{\breve{U}}(\breve{u})=\left\{\begin{array}{cc}
\frac{f_{U}(u)}{\int_{0}^{1} f_{U}(u) d u} & \text { si } u \leq 1 \\
0 & \text { en otro caso }
\end{array}\right.
$$

Como las variables $\mathrm{X}$ y $\mathrm{Y}$ son normales, entonces la integral $I=\int_{0}^{1} f_{w}(w) d w$ puede ser evaluada como la probabilidad $\mathrm{P}[\mathrm{X} \leq \mathrm{Y}]$, debido a que $\mathrm{Z}=\mathrm{Y}-\mathrm{X}$, entonces

$$
\mathrm{P}[\mathrm{X} \leq \mathrm{Y}]=\mathrm{P}[\mathrm{Y}-\mathrm{X} \leq 0]=\mathrm{P}[\mathrm{Z} \leq 0]
$$

La variable $\mathrm{Z}$ preserva la normalidad con media y varianza con $\mu_{\mathrm{z}}=\mu_{\mathrm{Y}}-\mu_{\mathrm{x}}$ y $\sigma_{\mathrm{Z}}^{2}=\sigma_{\mathrm{Y}}^{2}+\sigma_{\mathrm{X}}^{2}$. Por lo tanto:

$$
I=\int_{0}^{1} f_{U}(u) d u=P[Z \leq 0]=\Phi\left(\frac{0-\mu_{Z}}{\sigma_{Z}}\right)=\Phi\left(\frac{\mu_{X}-\mu_{Y}}{\sqrt{\sigma_{Y}^{2}+\sigma_{X}^{2}}}\right)
$$


Figura 2. Distribución probabilística del OEE

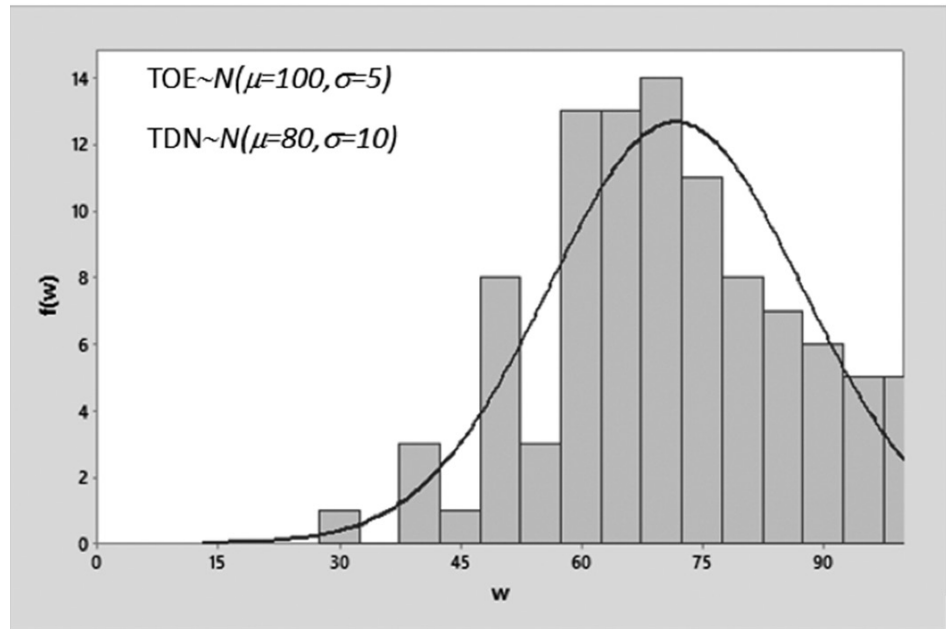

Fuente. Elaboración propia de los autores.

Por tanto, se puede tener una forma de la distribución para el OEE a partir de la ecuación (7), la figura 2 muestra la forma de la distribución probabilística del OEE.

Bajo este esquema se puede hacer una caracterización mejor del modelo. Lo primero que se considera es que el denominador TDN se puede obtener restándole al tiempo total disponible (Tiempo Calendario) el tiempo No Operacional TNO. EI TNO corresponde a los siguientes ítems:

- Mantenimiento planeado.

- Tiempo fuera de programación.

- Tiempo no programado por falta de recurso.

- Tiempo para pruebas y/o ensayos.

Todo tiempo que ha sido planeado fuera del programa de producción, aunque son conocidos y son específicos, tienen un comportamiento aleatorio por que en algunas semanas hay días festivos y en otras no, por lo tanto no todas las semanas se deja de programar los domingos, no siempre se programan 
los mismos turnos (por las necesidades de producción), no siempre se programan tiempos para inventarios y no en todas las áreas, se pueden programar "over hall" (mantenimiento completos de las líneas). Por lo tanto, se puede considerar que los TNO es una suma de tiempos aleatorios y por el teorema del límite central, se tiene que:

$$
\begin{gathered}
\mathrm{TNO}=\sum_{\mathrm{i}=1}^{\mathrm{N}_{1}} \mathrm{~T}_{\mathrm{i}} \sim \mathrm{N}\left(\sum_{\mathrm{i}=1}^{\mathrm{N}_{1}} \mu_{\mathrm{i}}, \sum_{\mathrm{i}=1}^{\mathrm{N}_{1}} \sigma_{\mathrm{i}}^{2}\right) \\
\operatorname{TDN}=\mathrm{TTD}-\sum_{\mathrm{i}=1}^{\mathrm{N}_{1}} \mathrm{~T}_{\mathrm{i}}
\end{gathered}
$$

Por otrolado, el numerador TOE que corresponde al tiempo operacional efectivo se puede expresar como TOE=TDNTTP-TPR-TPC. En la sección II se analizó que los tiempos de parada (TTP), los tiempos perdidos por rendimiento (TPR) y los tiempos perdidos por problemas de calidad (TPC), todos ellos son aleatorios, tanto en aparición de los eventos como en la duración de los tiempos, por esto se puede definir que:

$$
\begin{gathered}
\mathrm{TTP}+\mathrm{TPR}+\mathrm{TPC}=\sum_{\mathrm{j}=1}^{\mathrm{N}_{2}} \mathrm{~T}_{\mathrm{j}} \sim \mathrm{N}\left(\sum_{\mathrm{j}=1}^{\mathrm{N}_{2}} \mu_{\mathrm{j}}, \sum_{\mathrm{j}=1}^{\mathrm{N}_{2}} \sigma_{\mathrm{j}}^{2}\right) \\
\text { TOE }=\mathrm{TTD}-\sum_{\mathrm{i}=1}^{\mathrm{N}_{1}} \mathrm{~T}_{\mathrm{i}}-\sum_{\mathrm{j}=1}^{\mathrm{N}_{2}} \mathrm{~T}_{\mathrm{j}}
\end{gathered}
$$

De esta manera se puede expresar el OEE operacional en términos de suma de variables aleatorias, de la forma siguiente:

$$
\mathrm{OEE}=\frac{\mathrm{TOE}}{\mathrm{TDN}}=\frac{\mathrm{TTD}-\sum_{\mathrm{i}=1}^{\mathrm{N}_{1}} \mathrm{~T}_{\mathrm{i}}-\sum_{\mathrm{j}=1}^{\mathrm{N}_{2}} \mathrm{~T}_{\mathrm{j}}}{\mathrm{TTD}-\sum_{\mathrm{i}=1}^{\mathrm{N}_{1}} \mathrm{~T}_{\mathrm{i}}}
$$


Esto permite comprobar que realmente que el OEE es un cociente de suma de variables aleatorias (en frecuencia como en tiempo), y que por el teorema central del límite, se puede considerar que ellas tienen una distribución normal.

\section{Aplicación práctica del modelo estocástico de $\mathrm{OEE}$}

e hace una aplicación real en una línea de empaque farmacéutico que está integrada una máquina blíster y una estuchadora. El diagrama de proceso se describe en la figura 3.

Figura 3. Proceso de empaque farmacéutico tipo blíster

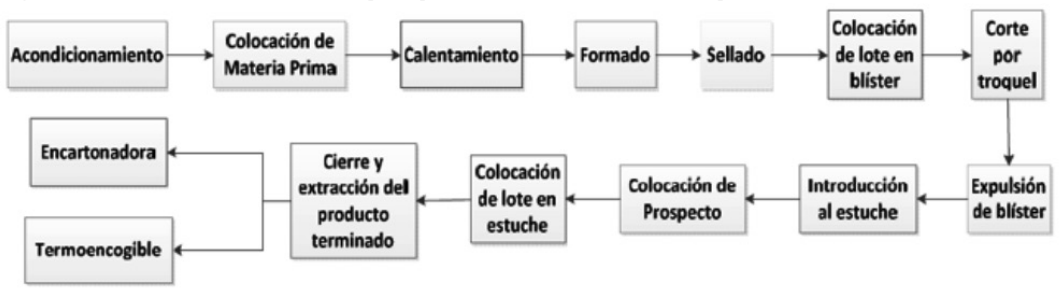

Fuente. Abrego, 2012.

Las líneas de proceso de empaque farmacéutico tiene múltiples etapas de proceso, que básicamente está divido en dos: el empaque primario (donde el empaque está en contacto directo con el producto, el blíster) y el empaque secundario, donde cada empaque primario (tiras de blíster) se imprime información exigida por normativa y se les coloca hologramas de seguridad y luego son empacados en cajas o estuches, y estos son empacados en cajas de cartón, generando la presentación final. Una característica que tienen es que el empaque primario se realiza en zona blanca (aislada y bajo 
condiciones de asepsia) y el empaque secundario se realiza en zona gris, donde no hay condiciones asepsia extrema.

Un elemento muy importante para el análisis, es el horizonte de gestión del OEE, este depende de la estructura operacional de las líneas, de la forma de gestión del proceso, de la planeación de producción y gestión del costeo. En caso de las líneas de empaque farmacéutico, por lo general se gestiona el proceso por órdenes de fabricación, que dependiendo del tipo y la demanda, pueden durar menos de un turno o más (y hasta días, dependiendo del tamaño del lote), por esta razón es que el horizonte de planeación y de gestión de la producción se realiza por semana. La aplicación considera el horizonte de gestión del OEE de una semana. La programación se realiza por turnos debido a esto se considera el tiempo disponible operacional como turnos (disponibles y utilizables desde la planeación), que son convertidos en unidades de gestión de horas.

Para el análisis de la aplicación del modelo estocástico del OEE propuesto, se tomaron los tiempos operacionales semanales de una línea de empaque farmacéutico, durante el año de 2014. Donde cada semana se registran los días utilizables, los festivos, los turnos utilizables y los reales programados así como tiempo operativo efectivo.

Para cada semana se hizo el cálculo del OEE2, para esto se midió el Tiempo Total Disponible por semana como $T_{T}=7$ días*24 horas=168 horas a partir de este tiempo se va restando los tiempos asociados a cada una de la pérdidas consideradas dentro del árbol de pérdidas (Sánchez, 2015). 
Figura 4. Comportamiento del OEE2

(a)

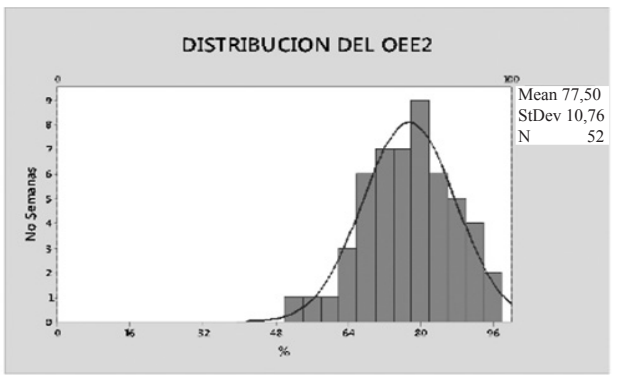

(b)

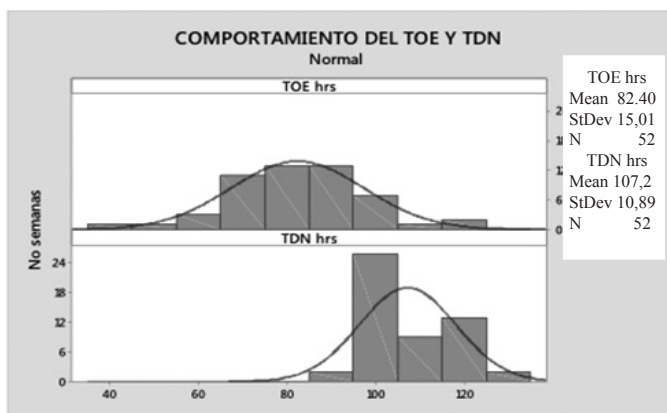

Fuente. Elaboración propia de los autores.

La figura 4(a) muestra el comportamiento estocástico del OEE2 semanal, lo primero que se observa es que el comportamiento es similar al del modelo propuesto e ilustrado en la figura 2, de la normal truncada. Lo importante es que se está mostrando la dispersión que tiene este OEE2, que puede fluctuar entre $49 \%$ y $96 \%$ y cuya desviación estándar muestral es de 10,26\%, que es bastante grande. Para completar el análisis de la estocasticidad del OEE, se hizo un análisis del comportamiento aleatorio del numerador TOE y el denominador TDN, la figura 4(b) muestra el comportamiento y se puede observar que tiene un comportamiento normal, que al hacer la prueba de ajuste se acepta significativamente.

Ahora se analiza el modelo en detalle, para esto se considera los tiempos perdidos, asociados al árbol de pérdidas las cuales permite clasificar estos tiempos por su naturaleza, hay tiempos perdidos por paradas programadas (Setups o 
cambios de insumo), tiempos perdidos operacionales no programados (Fallos de proceso, por problemas logísticos o problemas de calidad de ME), tiempos perdidos por fallas y averías de los equipos (averías mecánicas o electrónicas), y tiempos perdidos por rendimiento y calidad. La tabla 1 muestra los tiempos que se registraron y se agruparon de acuerdo al árbol de pérdidas de la línea de empaque, definida previamente.

Tabla 1. Datos de pérdidas para el cálculo del OEE

\begin{tabular}{|c|c|c|c|c|c|c|c|c|c|c|c|c|c|c|}
\hline $\begin{array}{c}\text { No } \\
\text { SEMANA }\end{array}$ & TOE & Inicio/Fin & Setups & $\begin{array}{l}\text { Cambio } \\
\text { Insumo }\end{array}$ & $\begin{array}{l}\text { Interrucion } \\
\text { Programada }\end{array}$ & Averias & $\begin{array}{c}\text { Fallos } \\
\text { Externos }\end{array}$ & $\begin{array}{c}\text { Fallos } \\
\text { Proceso }\end{array}$ & $\begin{array}{l}\text { Problemas } \\
\text { Calidad ME }\end{array}$ & $\begin{array}{l}\text { Problemas } \\
\text { Logisticos }\end{array}$ & $\begin{array}{l}\text { Problemas } \\
\text { planeación }\end{array}$ & $\begin{array}{c}\text { Organización } \\
\text { Gestion }\end{array}$ & Rendimiento & Calidad PT \\
\hline 1 & 42,2 & 0,8 & 6,7 & 0,2 & 1,2 & 6,3 & 2,1 & 10,6 & 3,0 & 3,8 & 0,6 & 2,5 & 3,4 & 5,1 \\
\hline 2 & 71,8 & 1,4 & 12,7 & 0,4 & 0,7 & 10,8 & 3,6 & 17,9 & 5,0 & 6,5 & 1,1 & 4,3 & 5,7 & 8,6 \\
\hline 3 & 75,6 & 1,5 & 13,4 & 0,4 & 0,8 & 11,3 & 3,8 & 18,9 & 5,3 & 6,8 & 1,1 & 4,5 & 6,0 & 9,1 \\
\hline 4 & 53,0 & 1,1 & 8,9 & 0,3 & 0,5 & 7,9 & 2,6 & 13,2 & 3,7 & 4,8 & 0,8 & 3,2 & 4,2 & 6,4 \\
\hline 5 & 75,3 & 1,5 & 13,4 & 0,4 & 0,8 & 11,3 & 3,8 & 18,8 & 5,3 & 6,8 & 1,1 & 4,5 & 6,0 & 9,0 \\
\hline 6 & 96,1 & 1,9 & 17,5 & 0,5 & 1,0 & 14,4 & 4,8 & 24,0 & 6,7 & 8,6 & 1,4 & 5,8 & 7,7 & 11,5 \\
\hline 7 & 87,3 & 1,7 & 15,8 & 0,4 & 0,9 & 13,1 & 4,4 & 21,8 & 6,1 & 7,9 & 1,3 & 5,2 & 7,0 & 10,5 \\
\hline 8 & 86,9 & 1,7 & 15,7 & 0,4 & 0,9 & 13,0 & 4,3 & 21,7 & 6,1 & 7,8 & 1,3 & 5,2 & 7,0 & 10,4 \\
\hline 9 & 78,2 & 1,6 & 13,9 & 0,4 & 0,8 & 11,7 & 3,9 & 19,5 & 5,5 & 7,0 & 1,2 & 4,7 & 6,3 & 9,4 \\
\hline 10 & 56,5 & 1,1 & 9,6 & 0,3 & 0,6 & 8,5 & 2,8 & 14,1 & 4,0 & 5,1 & 0,8 & 3,4 & 4,5 & 6,8 \\
\hline 11 & 107,9 & 2,2 & 19,9 & 0,5 & 1,1 & 16,2 & 5,4 & 27,0 & 7,6 & 9,7 & 1,6 & 6,5 & 8,6 & 12,9 \\
\hline
\end{tabular}

Fuente. Elaboración propia de los autores.

Para cada grupo de pérdidas incluidas en la tabla 1, se ajustó el modelo Weibull propuesto dentro del modelo. Primero se hizo los ajustes para los tiempos perdidos por paradas programadas, en la figura 5 se muestran los ajustes a la distribución Weibull, de los tiempos de: arranques y despejes finales; setups o cambios de referencia; cambio de insumos (rollos) y las interrupciones programadas (limpieza). De esta forma se tiene que las distribuciones de cada tiempo tienen los siguientes ajustes:

$$
\begin{gathered}
\mathrm{T}_{\text {Iniciofin }} \sim \text { Weibull(1.7,6); } \mathrm{T}_{\text {Setups }} \sim \text { Weibull }(5.4,16) ; \\
\mathrm{T}_{\text {CInsumo }} \sim \text { Weibull }(0.4,6) ; \mathrm{T}_{\text {IntPrograma }} \sim \text { Weibull }(0.9,5.9) .
\end{gathered}
$$


Figura 5. Ajustes de los tiempos programados rutinarios

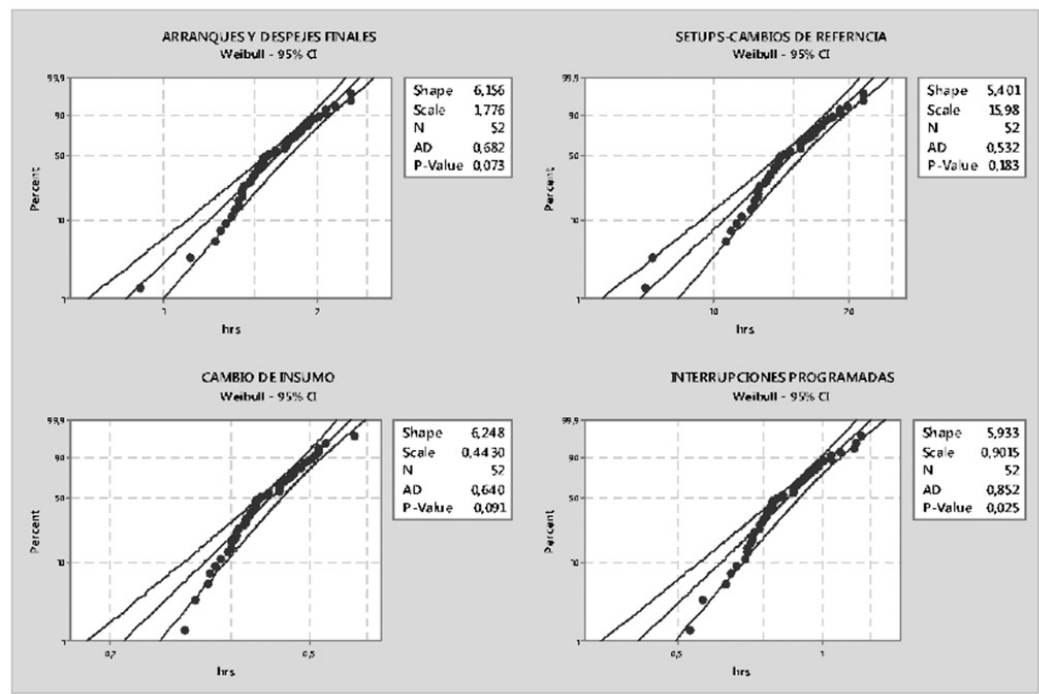

Fuente. Elaboración propia de los autores.

Para las paradas inesperadas operacionales, se incluyen los tiempos perdidos por: Fallos Externos (equipos críticos y de apoyo), Fallos de proceso (Ajustes de proceso y malla reventada), por Problemas de Calidad (ME defectuosos, Tabletas/Cápsulas fuera de especificación), Por problemas Logísticos (Retraso en dispensación de Materiales tanto de MP como ME), por Problemas de Planeación (cambio de programa, falta de materiales adicionales) y por problemas de gestión y organización (Falta de operarios, documentación, espera por análisis y disposición de aseguramiento de calidad). Los ajustes se muestran en la figura 6 y todos los tiempos se ajustan a una distribución Weibull. Así las distribuciones de cada tiempo perdido es:

$$
\begin{aligned}
& \mathrm{T}_{\text {FallosEx }} \sim \text { Weibull(1.4,6.1) } \quad \mathrm{T}_{\text {Proceso }} \sim \text { Weibull(4.2,18.8) } \quad \mathrm{T}_{\text {CalidadME }} \sim \text { Weibull(5.9,7), } \\
& \mathrm{T}_{\text {PLogist }} \sim \text { Weibull(8.4,5.5), } \mathrm{T}_{\text {PPlanea }} \sim \text { Weibull(1.4,2.7) } \quad \mathrm{T}_{\text {OrgGestion }} \sim \text { Weibull }(1.7,3.5)
\end{aligned}
$$

De la misma forma se analizaron los tiempos perdidos por averías, para esto se estudiaron de dos formas, los tiempos perdidos por averías semanal (horizonte de análisis) y también 
por averías individuales, la figura 7 muestran el ajuste de los dos tiempos, y muestran que tienen un comportamiento Weibull.

De esta forma se puede concluir que los tiempos son Weibull:

$$
\mathrm{T}_{\text {Averías-semanal }} \sim \text { Weibull(13.29,6); } \mathrm{T}_{\text {Averias }} \sim \text { Weibull(1.84,1.1); }
$$

Figura 6. Ajustes de los tiempos inesperados operacionales

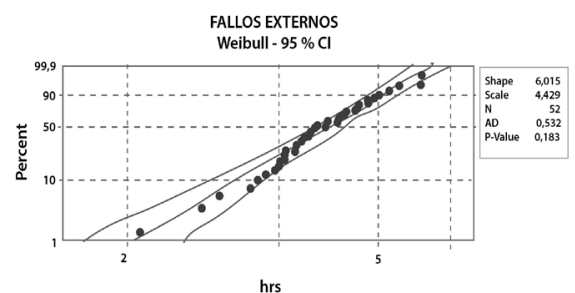

hrs

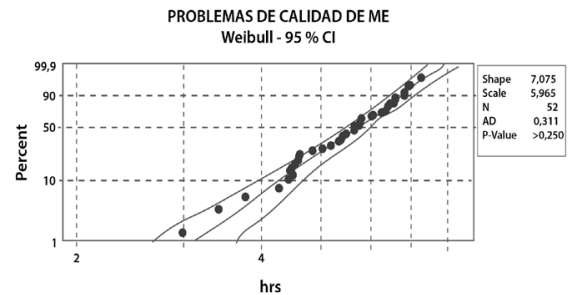

PROBLEMAS DE PLANEACIÓN Weibull - $95 \% \mathrm{Cl}$

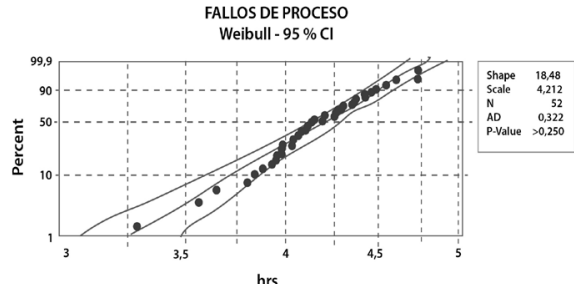

PROBLEMAS LOGÍSTICOS Weibull $-95 \% \mathrm{Cl}$

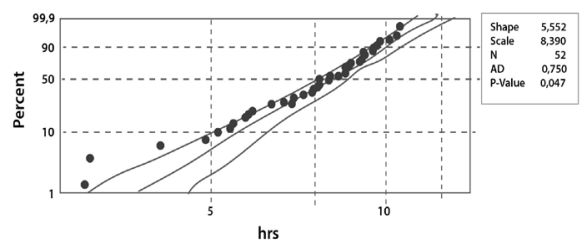

PROBLEMAS DE ORGANIZACIÓN Y GESTIÓN Weibull - $95 \% \mathrm{Cl}$

Fuente. Elaboración propia de los autores.

Este modelo es lógico porque como se analiza en análisis de confiabilidad y mantenibilidad, el modelo que generalmente se estudia es de Weibull.

Se puede tener estimaciones de cuál sería el OEE2 promedio estimado para el proceso, en forma general (futuro) usando la simulación a través de Bootstrap (remuestreo), de la misma forma para la desviación estándar (Sánchez, 2015), y a partir de allí se pueden hacer inferencias 
estadísticas, pudiéndose hacer análisis mas profundos del comportamiento probabilístico del OEE.

Figura 7. Ajustes de los tiempos perdidos por averías.
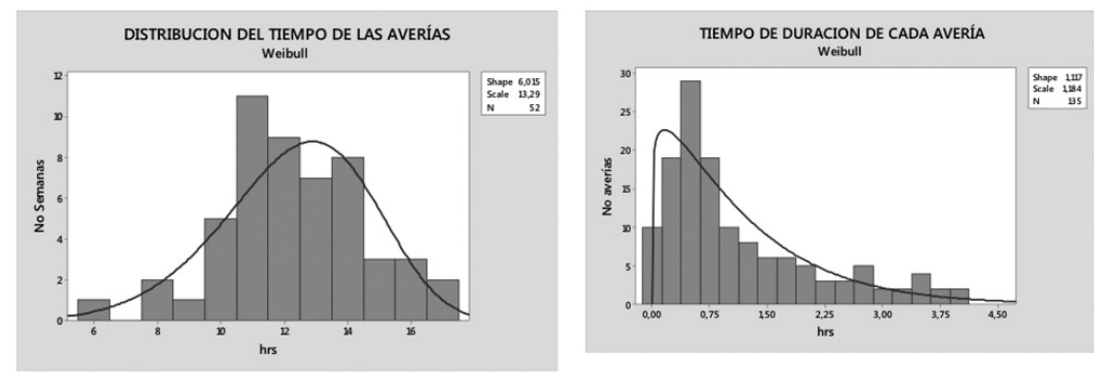

Fuente. Elaboración propia de los autores.

\section{Consideraciones prácticas del modelo estocástico del OEE}

n esta sección se analiza la utilidad práctica de disponer de un modelo estocástico del OEE, y especialmente para dar valor agregado en la toma de decisiones con respecto a la eficiencia global de las líneas o equipos. Hasta el momento se ha propuesto un modelo estocástico para el OEE y se ha mostrado una aplicación de cómo utilizarlo, pero el análisis no se puede quedar ahí, porque de nada sirve disponer un modelo si no se discute cuál es su utilidad práctica y como puede dar un valor agregado en la toma de decisiones con respecto a la eficiencia global de las plantas y de los equipos. 
Desde el punto de vista general, disponer de un modelo como éste puede ser una herramienta muy robusta de análisis y evaluación de toma de decisiones. El mejor símil que se puede hacer es, con los modelos econométricos que describen las relaciones y los comportamientos de un sistema económico, con ellos se pueden simularse escenarios de política económica, pudiéndose pronosticar y evaluar comportamientos futuros bajo restricciones de los escenarios planteados, de esta forma evaluar intervenciones del sistema en forma proactiva y no esperar a intervenir el sistema para evaluar el resultado (en forma reactiva) y allí hacer los ajustes. De la misma forma, se podría hacer con el OEE para evaluar mejoras y decisiones en forma preventiva.

Aquí solo se mencionan algunas de las posibles aplicaciones prácticas del modelo estocástico (en Sánchez 2015, se hace un análisis más detallado):

- Aplicación en la optimización de los setups: Normalmente cuando se quiere optimizar los setups (cambios de referencia), se utiliza la metodología SMED (Shingo, 1998). Aquí se puede plantear un mejora relacionada con reducir los tiempos de cambio de referencia, pero es muy importante primero formular objetivos de mejora y cuantificar cuál puede ser el impacto de reducir estos tiempos sobre el OEE, estimación que puede realizarse con el modelo. Por otro lado, también se puede evaluar cuál es el impacto de la mejora sobre la dispersión; a través del modelo y del análisis detallado de las pérdidas se puede entrar a analizar especialmente aquellos tiempos de setups altos y que en ocasiones son atípicos, como una estrategia de estandarización y reducción de la dispersión, se puede hacer una simulación, usando el modelo y las estimaciones hechas para cada tiempo perdido que componen el OEE, con el fin de evaluar cuánto sería su impacto sobre la dispersión del OEE y lo más importante, evaluar el nivel 
de mejora que puede llegar a lograrse y así evaluar su impacto económico con anterioridad. En Sánchez (2015) se hace un aplicación de esta consideración se muestra el impacto de las posibles mejoras, centrándose en un solo tipo de referencia o en varias, de las misma forma se pudo analizar el impacto desde el punto de vista de reducción de dispersión.

- Aplicación a la minimización de los tiempos perdidos por averías. Otra pérdida que por lo general aparece dentro del Pareto de tiempos perdidos y que impactan a la disponibilidad, es el de las averías. A pesar de que el análisis de averías es un problema muy complejo, que por lo general se abordan en el pilar de mantenimiento planificado del TPM, implica que tiene que hacer análisis más profundos y hacer intervenciones donde se involucran aspectos tanto técnicos como humanos, pero la práctica dice que lo más impactante es la política de mantenimiento que se tenga. En particular, se puede utilizar el modelo estocástico de OEE para evaluar por ejemplo, cual sería el impacto de pasar de una política de reemplazar piezas o componentes críticos "correctiva", es decir, sustituirlas solo cuando fallan; frente a una política de reemplazo preventivo, es decir reemplazarlas en tiempo fijo, antes de que falle. Este aspecto es muy importante porque, disponer la posibilidad de evaluar el cambio de la política y su impacto sobre el OEE, a través de simulación. Sánchez (2015) ilustra una aplicación de cómo se puede realizar esto.

- Aplicación de la reducción de la variabilidad del OEE. Cuando se hace un análisis de pérdidas por disponibilidad es muy importante hacer el análisis del comportamiento de la dispersión del OEE, como se observa en la figura 4, el OEE promedio es $77,5 \%$ con una desviación del 10,3\%, dado un coeficiente de variación del $13 \%$, este es muy alto, 
eso quiere decir que el OEE no es homogéneo. Si se quiere, atacar este problema, (la reducción de dispersión), se debe hacer un análisis de Pareto de la variabilidad, para lo cual se requiere establecer cual pérdida es la que aporta más a la variabilidad, a través del coeficiente de contribución de la varianza de cada pérdida a la varianza total.

Figura 8. Pareto de contribuciones de causales de varianza

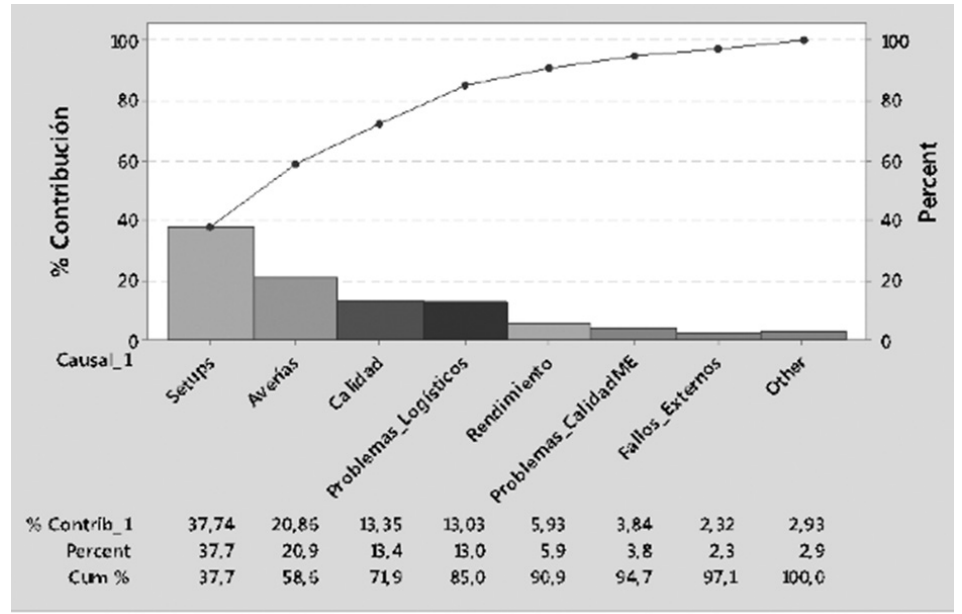

Fuente. Elaboración propia de los autores.

La figura 8 muestra la contribución de cada tipo de perdida, a la varianza total, la propiedad utilizada es la aditividad de la varianza, Sánchez (2015) ilustra el método para hacerlo. Allí se puede observar que los tiempos de setups y las averías son las pérdidas que más impactan $(48 \%)$ a la variabilidad del OEE, por lo tanto permite entrar a focalizar necesidades de mejora en términos de variabilidad, de hacer más homogéneo el OEE, y así estandarizar las pérdidas. En este caso, se debe desarrollar un proceso metodológico para hacer la reducción de la variabilidad del OEE. 


\section{Conclusiones}

- I modelo estocástico del OEE propuesto, está disponible para empezar a utilizarlo en aplicaciones prácticas y en especial para hacer análisis más formales para la toma de decisiones. Se plantea que se debe empezar a utilizar, para investigar y mostrar en profundidad el potencial y análisis más robustos que permiten tomar decisiones más fiables

La primera reflexión, que deja este análisis es que las personas responsables de los procesos de implementación de mejoras, de mantenimiento y de los programas de excelencia operacional, deben aprender más acerca del comportamiento del OEE y analizarlo desde la perspectiva de la variabilidad. A partir de allí, conocer cómo puede usarse para hacer análisis previos a las mejoras, haciendo simulaciones, permitiendo evaluar en forma cuantitativa las acciones propuestas de las mejoras y en especial, medir el efecto acumulado y global sobre el OEE y, por lo tanto sobre la eficiencia global del equipo. De esta forma se eliminan los paradigmas para la gestión de los procesos y las líneas, que se tienen para poder implementar los programas de excelencia operacional.

Es importante señalar que la gestión del OEE en forma práctica no se puede hacer aislado del entorno, o través de la gestión del frío número. Este análisis debe estar ligado al análisis del Árbol de Pérdidas, porque con este se puede mapear el comportamiento de las ineficiencias de la organización y este da una visión más global y sistémica de los problemas dentro de la organización. Y así, poder mirar la excelencia como una estrategia global de organización y realizar el despliegue desde lo más estratégico a lo más operativo, comprendiendo, de una forma proactiva y sistémica, la dinámica y la problemática operacional, con el 
fin de lograr proponer mejoras de gran impacto y de alta duración que perduren. El aspecto nuevo que propone el artículo es la aplicación práctica del modelo matemático propuesto y las posibilidades de ampliación del análisis, complementándolo con el árbol de pérdidas. 


\section{Referencias bibliográficas}

Abrego M. F. (2012). Determinación de las condiciones óptimas del procedimiento del material primario en las maquinas emblistadoras. Proyecto de grado Ingeniería de Materiales. Venezuela: Universidad Simón Bolívar.

Álvarez H. (2010). El Árbol de pérdidas y TPM. Recuperado de: www.ceroaverias.com.

Farnum N. R. \& Stanto L (1987). Some Results Concerning the Estimation of Beta Distribution Parameter en PERT. The Journal of the Operational Research Society Vol 38 No 3 pp: 287-290.

Gouvea da Costa, SE. Pinheiro de Lima, E. (2002). Uses and misuses of the overall equipment effectiveness for production management. IEEE International Engineering Mana-gement Conf. Cambridge, UK pp. 816-20.

Law A. M. \& Kelton W. D. (2000). Simlation Modeling and Analysis. McGraw Hill.

Ljunberg, Ö. (1998). Measurement of overal equipment as a basis for TPM activities. International Journal of Operations \& Production Managment. Vol 18, No 5. pp. 495-507.

Nakajima, S. (1988). Introduction to TPM. Cambridge, MA Productivity Press.

Roussas, G. G. (1997). A Course in Mathematical Statistics. 2d Edition. London: Academic Press. 
Sánchez R. A. (2015). Construcción de un modelo estocástico para la Eficiencia Global de los Equipos. Tesis de Maestría. Ingeniería Industrial. Bogotá, Colombia:éUniversidad DistritaL Francisco Jose de Caldas.

SEMI Semiconductor Equipment and Materials International (1992). SEMI Standars: Equipment Automation/Harware Volume, Semiconductor Equipment and Materials International. Mountain View, CA.

Stamatis D. H. (2010). The OEE Primer: Undestanding Overall Equipment Effectiveness, Reliability and Mantenibility. New York: CRC Press.

Womack J. P. \& Jones D. P. (2012). Lean Thinking. 2a Edición. España: Ediciones Gestión 2000.

Yacuzzi, E., Arancio, E., Alfonso, D., Esteche, M. H \& Niro, V. (2011). Como realizar un Plan Hoshin: una aplicación en ASC. Telesi S.R.L. Documento de trabajo, caso de negocios. Buenos Aires Argentina. Universidad CEMA.

Zammori F., Braglia, M. \& Frosolini, M. (2011). Stichastic Overall Equipment Effectiveness. International Journal of Production Research. Vol 49 No 21. pp: 1-41. 